\title{
The stellar kinematics of the giant halos of Brightest Cluster Galaxies
}

\author{
George K. T. Hau ${ }^{1} \dagger$, M. Hilker ${ }^{2}$, T. Bridges ${ }^{3}$, D. Carter ${ }^{4}$, \\ H. Dejonghe ${ }^{5}$, S. De Rijcke ${ }^{5}$ and H. Quintana ${ }^{6}$ \\ ${ }^{1}$ European Southern Observatory, Karl-Schwarzschild-Str. 2, D-85748, Garching-bei-München, \\ Germany email: ghau@eso.org \\ ${ }^{2}$ Sternwarte der Universität Bonn, Auf dem Hügel 71, D-53121, Bonn, Germany \\ ${ }^{3}$ Department of Physics, Queen's University, Kingston, Ontario, K7L 3N6, Canada \\ ${ }^{4}$ Astrophysics Research Institute, Liverpool John Moores University, L41 1LD, UK \\ ${ }^{5}$ Department of Mathematical Physics and Astronomy, Ghent University, B-9000 Belgium \\ ${ }^{6}$ Facultad de Física y Astronomía, Universidad Católica de Chile, Santiago, Chile
}

\begin{abstract}
We have investigated the stellar kinematics of two brightest cluster galaxies (BCGs) to large distances from the cluster centre. In both galaxies, the velocity dispersion rises with radius. Orbital anisotropy is ruled out as the cause and we conclude that the kinematics in the outer parts of BCGs are dark matter dominated. This result contrasts with the current problem for intermediate luminosity ellipticals, which appear to have less dark matter than predicted by CDM. Combining the present results with our previous work, the fraction of BCGs with outwardly rising dispersions $(\sim 60 \%)$ is much higher than that found previously $(\sim 7 \%)$ from the inner parts of BCGs. We argue that this phenomenon may be more common than previously thought. The agreement with radial trends expected for a smooth galaxy/cluster transition, and the lack of dependence on $\mathrm{cD} / \mathrm{BCG}$ dominance and cluster environment, suggest that the outwardly rising dispersion is a genuine response of the stars to the underlying cluster potential. There is evidence that BCG in Abell 2063 is not at the centre of the cluster potential.
\end{abstract}

\section{The "dearth of dark matter" problem in intermediate luminosity ellipticals}

Are elliptical galaxies good places to look for dark matter? In the Cold Dark Matter (CDM) paradigm, elliptical galaxies are expected to possess a massive dark halo made up of subhalos of hierarchically merged galaxies, and their kinematics in the outer parts are expected to be dominated by the dark matter potential. Recent observational results on intermediate-luminosity (and mass) ellipticals seem to challenge this assumption. Measurements, using Planetary Nebulae, of the radial dispersion profiles of 3 intermediateluminosity ellipticals to large radii show that they decrease with distance from the galaxy centre, with little evidence of dark matter (Romanowsky et al. 2003). The combined kinematics of the stars and $\mathrm{PNe}$ can be described well by constant $M / L$ ratio models, with $M / L$ lower than CDM predictions and consistent with a stellar $M / L$ ratio. For one of these galaxies (NGC 3379), the globular cluster kinematics also support this finding (Bridges et al. 2003, Beasley et al. 2004 in preparation). This so-called "dearth of dark matter" problem is one of the current challenges for CDM (Binney 2004).

$\dagger$ Current address: Department of Physics, Durham University, South Road, Durham DH1 3LE, UK 


\section{Dynamical evidence for dark matter in luminous ellipticals/BCGs}

While the dynamical influence of dark matter in spirals is evident from their flat rotation curves, it is not so obvious in ellipticals, as they are pressure supported and the anisotropy parameter is unknown. For luminous ellipticals and BCGs, direct dynamical evidence comes from their flat dispersion profiles (e.g. Bertin et al. 1994, Carollo et al. 1995, Fisher et al. 1995, FIF95, Saglia et al. 1993, 2000, Kronawitter et al. 2000), and outwardly rising dispersion profiles (e.g. Dressler 1979, Carter et al. 1985, 1999, CBH99, Kelson et al. 2002) have been observed for a few galaxies. The latter is one of the strongest signatures of the dominance of dark matter, but as with the other cases is still affected by the degeneracy between orbital anisotropy and $M / L$ : an increase in velocity dispersion may be due to an increase in $M / L$, or alternatively due to an increasing bias towards tangential orbits (i.e. more stars moving along the line-of-sight; see e.g. Carter et al. 1985). Thus only in a few cases where information on the orbital anisotropy is available can the alternative be ruled out.

Information on the orbital anisotropy may come from the shape of the Line-of-Sight Velocity Distributions (LOSVDs) - in the outer parts of the galaxy tangential orbits should produce flat-topped profiles, whereas a bias towards radial orbits should produce pointy profiles (van der Marel \& Franx 1993, vdM93). However, due to the extremely faint nature of BCG halos, only in a few examples have the line-profiles been included in the kinematic measurements (e.g. CBH99, Saglia et al. 2000, Kronawitter et al. 2000). The availability of $8-\mathrm{m}$ class telescopes have made it possible to measure not just the velocity and dispersion but also the Gauss-Hermite moments $h_{3}$ and $h_{4}$ of the LOSVDs to large distances from the galaxy centre. The $h_{4}$ parameter is especially sensitive to tangential anisotropy (vdM93).

We have embarked on a program to study the dynamics and stellar populations of BCGs. Here we present results on two BCGs observed with FORS1 in long-slit mode on the VLT, representing one of the deepest studies to date.

\section{The sample and results}

Abell 2063 is part of the Hercules Supercluster, and has intermediate cD dominance, with a Bautz-Morgan classification of II. It has dynamical subclumps and the central BCG (CGCG 077-097) has a peculiar velocity with respect to the cluster, which may indicate merger (Girardi et al. 1997, Krempeć-Krygier \& Krygier 1999). Abell 1060/Hydra $\mathrm{I}$ is a dynamically evolved cluster with a regular core shape and isothermal X-ray gas halo. With Bautz-Morgan classification of III, the domination of the central cD (NGC 3311 ) is insignificant. The total exposure times along the major axes of the two galaxies are 3.5 and $5 \mathrm{hr}$ respectively.

Kinematic analysis was performed using van der Marel's code (vdM93). The GaussHermite parameters $v, \sigma, h_{3}$ and $h_{4}$ were derived from spectra with $S / N \approx 35$. For the outer parts of Abell 2063, spectra with $S / N \approx 20$ are used to derive $v$ and $\sigma$ only. The outermost datapoints in Fig. 1 correspond to distances of $7.9 \mathrm{kpc}$ and $5.5 \mathrm{kpc}$ for Abell 1060 and Abell 2063 respectively $\left(\mathrm{H}_{0}=70 \mathrm{~km} \mathrm{~s}^{-1} \mathrm{Mpc}^{-1}\right)$. In Fig. 1 we present radial profiles of the kinematic parameters. The velocity dispersions in both galaxies rise outwardly. The $h_{4}$ values in the outer parts of the galaxy show that the LOSVD is not flat-topped, and that the rise in dispersion does not have a corresponding increase in tangential anisotropy which will lower the $h_{4}$. In combination with the surface brightness profile of the stellar population, it shows that the dynamics of the stars in the outer parts of the BCGs are dominated by the dark matter potential. 

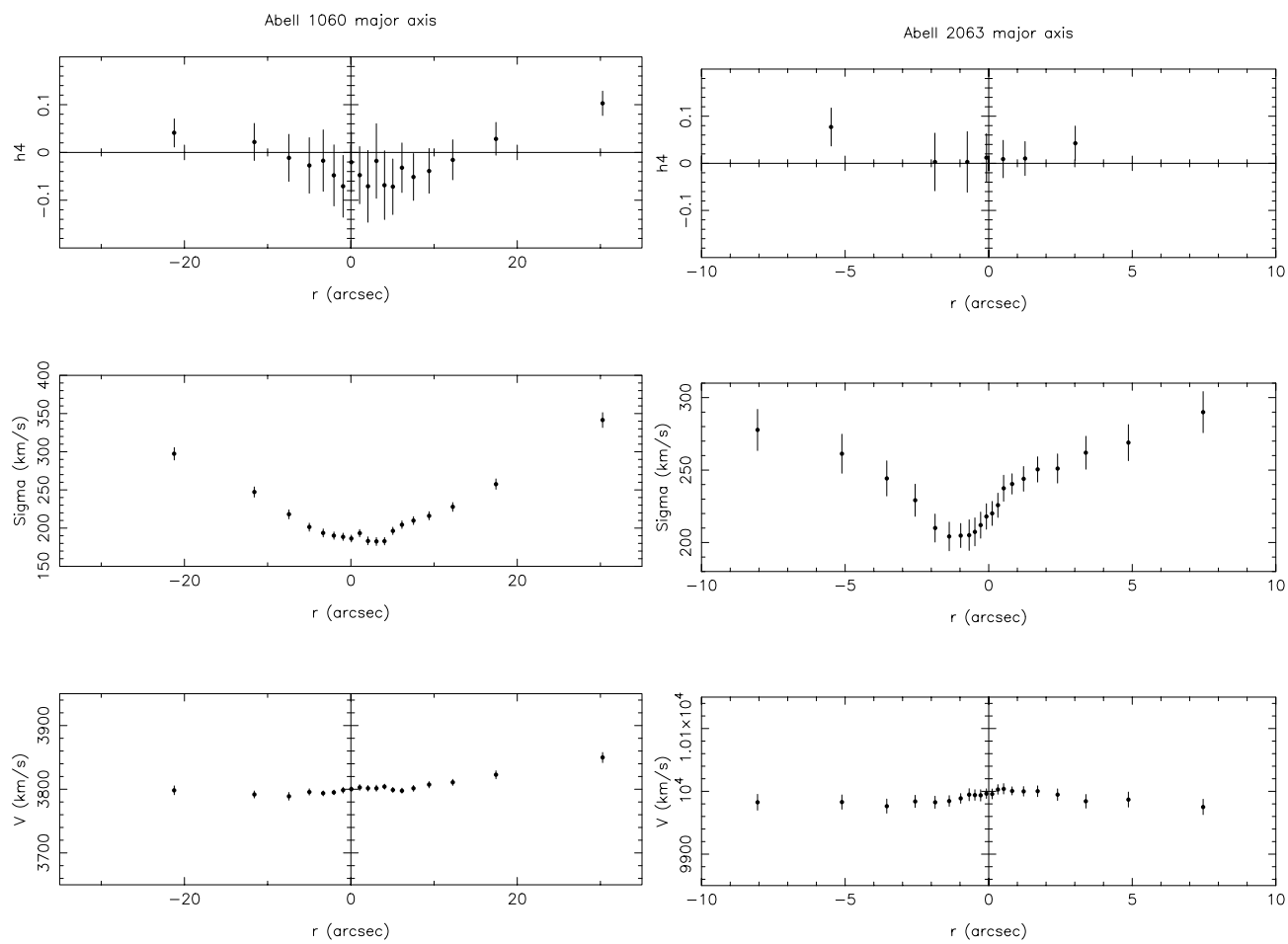

Figure 1. The mean velocity $v$, velocity dispersion $\sigma$, and Gauss-Hermite parameter $h_{4}$ of the BCGs in Abell 1060 (left) and Abell 2063 (right) as a function of distance $r$ from the nucleus along the major axes. A positive $h_{4}$ indicates a LOSVD more pointy than a Gaussian, whereas a negative $h_{4}$ indicates a flat-topped LOSVD. The fact that the increase in $\sigma$ has no corresponding decrease in $h_{4}$ shows that a bias towards tangential orbits is not responsible for the outwardly rising $\sigma$ profile.

For Abell 2063, the minimum in the velocity dispersion is displaced about $1^{\prime \prime}(0.7 \mathrm{kpc})$ south of the centre in the light distribution, suggesting that the BCG is offset from the centre of the cluster potential. Although a $V-I$ map shows a nuclear region about $0.8^{\prime \prime}$ in size and $\sim 0.04 \mathrm{mag}$ redder than the surroundings, it is unlikely that the presence of dust has affected the determination of the galaxy centre for two reasons. Firstly, the centre of the light distribution is obtained by a fit to the light profile inside the half light radius and is thus insensitive to the peak, and secondly the dust feature coincides with the centre of the light distribution. An X-ray map shows that the BCG is situated at the peak of the cluster X-ray distribution, but does not have high enough spatial resolution to resolve a $1^{\prime \prime}$ offset (Jones \& Forman 1999). The possibility that the BCG of Abell 2063 is not at the minimum of the cluster potential has also been noted by others (Girardi et al. 1997, Krempeć-Krygier \& Krygier 1999).

In Fig. 2 we plot the velocity dispersions against radius in log scale. Plotted also are the velocity dispersions of the cluster galaxies in different radial bins. For Abell 1060 they are calculated by searching in NED and HYPERLEDA for galaxies in different annuli and visually rejecting outliers in the velocity histograms. For Abell 2063, they are taken from Krempeć-Krygier \& Krygier (1999). For both galaxies, the rises in the stellar dispersions follow the trend expected if they are to join with those of the galaxies. This shows that, as the distance from the cluster centre increases, the stars are more and more influenced by the overall potential of the cluster than by the central BCG. 

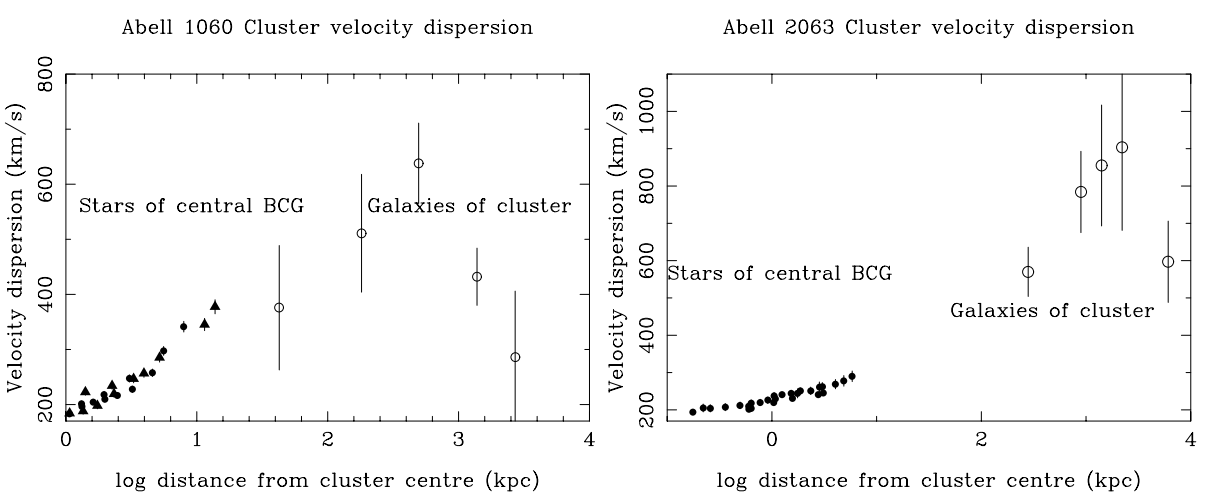

Figure 2. The major axis stellar velocity dispersions $\sigma$ of the BCGs in Abell 1060 (left) and Abell 2063 (right) against log radius (solid circles). Plotted also are the velocity dispersions of the cluster galaxies in different radial bins (open circles). For Abell 1060, data along a line joining NGC 3311 and NGC 3309 is also available, and the dispersion values are indicated by the solid triangles. For Abell 2063, a -1" offset to the galaxy centre has been introduced.

\section{Discussions and conclusions}

In a previous study of BCG kinematics, only 1 out of 15 was found to have a rising dispersion profile (FIF95), and hence they were thought to be rare. Of the 5 galaxies studied by this collaboration (CBH99 and this work), we have found 3 with such features - a significant fraction. $\dagger$ Conceptually, if the stars and galaxies are dynamically relaxed, since they are moving in the same potential, the dispersion of the BCG is expected to rise outwards eventually and join smoothly with that of the cluster. It may be possible that others will show rising dispersion profiles given deep enough observations. Scaling mirror sizes, the equivalent exposure times for this work and Abell 2199 in CBH99 are 32 and 8 times that of FIF95 respectively. The FIF95 result for Abell 2199 is actually consistent with that of CBH99 as the velocity dispersion only starts rising beyond the outermose datapoint of FIF95. We argue that rising dispersion profiles are more common in BCGs than previously thought.

The occurrence of outwardly rising dispersion profiles seems not to be dependent on cD/BCG dominance. The two galaxies studied in this work have BM type II and III, and Abell 2199 in CBH99 has BM type I. The other 3 BCGs (Abell 2029 and 3266 and AS 0463) with rising dispersion profiles (albeit without constraints on orbital anisotropy) have BM types I, I-II and I-II respectively. Thus, although rising dispersion profiles tend to occur in clusters in which the $\mathrm{cD} / \mathrm{BCG}$ dominates, they also occur in clusters not dominated by the BCG. All the clusters are rich to moderately rich (richness class 2 to 1 ), but this may be a bias due to the lack of very rich clusters in the nearby Universe, and to the lack of $\mathrm{cD} / \mathrm{BCG}$ in poor clusters.

Outwardly rising dispersion profiles occur not only in seemingly relaxed clusters (Abell 1060 and 2199), but also in clusters with substructures (Abell 2063) and also in those with dumbbell morphology indicating ongoing collisions (Abell 3266). The insensitivity to the $\mathrm{cD}$ dominance and to the cluster environment lends support to the idea that the rising dispersion profiles indicate a genuine response of the stars to the underlying cluster potential, and that BCGs are dark matter dominated, in contrast to intermediate mass ellipticals. The caveat is that some seemingly relaxed clusters may turn out to be disturbed (e.g. Girardi et al. 1997).

$\dagger$ Note that CBH99 utilized a 2.5m telescope and Abell 2162 and 2197, which don't show an outwardly rising dispersion, have also the shortest exposure times of all 5 galaxies. 
U-shaped velocity dispersion profiles have also been seen in pairs of elliptical galaxies with dumbbell morphology (Madejsky 1991, Madejsky, Bender \& Moellenhoff 1991, Madejsky \& Bien 1993). However, in such systems the $v$ and $\sigma$ profiles are often asymmetric, and there may be significant rotation present. Although Abell 1060 (NGC 3311) is not classified as dumbbell, NGC 3309 is only 1.7' away. However, there is no significant rotation in NGC 3311, and symmetry is observed in both $v$ and $\sigma$ along two almost perpendicular axes. Fig. 2 also shows that the rising dispersion profiles are identical along the two axes. This further reinforces the idea that the rising dispersion profiles are genuinely caused by the underlying cluster potential

The contrast between BCGs and intermediate-mass ellipticals poses an interesting question: is dark matter depleted in intermediate-mass ellipticals, or distributed at much larger radii ( $>5 \mathrm{R}_{\text {eff }}$ ), as in possible scenarios to save CDM (Romanowsky et al. 2003)? Or is it because the privileged positions of BCGs, in the potential wells of massive clusters, make them the most extreme examples of dark matter domination? A study of ellipticals in intermediate environments and masses may help to resolve this issue.

We have presented qualitative interpretation of the observed kinematics. Detailed dynamical modelling will help to test the universality of the NFW profile. These results shall be presented in a future paper (Hau et al. in preparation).

\section{Acknowledgements}

We thank R. van der Marel for providing his code for kinematic measurements. We also acknowledge discussions with P. Lynam, N. Napolitano and E. Valentijn.

\section{References}

Bertin G., Bertola F., Buson L.M., Danzinger I.J., Dejonghe H., Sadler E.M., Saglia R.P., de Zeeuw P.T., Zeilinger W.W., 1994, A\&A, 292, 381

Binney J., 2004, in IAU Symposium 220, "Dark Matter in Galaxies", ed. S. Ryder, D.J. Pisano, M. walker \& K. Freeman, Publ. Astron. Soc. Pac.

Bridges T., Beasley M., Faifer F., Forbes D., Forte J., Gebhardt K., Hanes D., Sharples R., Zepf S. 2003, In Joint Discussion \#6: Extragalactic Globular Clusters and their Host Galaxies" (ed. T. Bridges \& D. Forbes). IAU General Assembly, July 2003, astro-ph/0310324

Carollo C. M., de Zeeuw P. T., van der Marel R. P., Danziger I. J., Qian E. E., 1995, ApJ, 441, L25

Carter D., Inglis I., Ellis R. S., Efstathiou G., Godwin J. G., 1985, MNRAS, 212, 471

Carter D., Bridges T. J., Hau G. K. T., 1999, MNRAS, 307, 131

Dressler A., 1979, ApJ, 231, 659

Fisher D., Illingworth G., Franx M., 1995, ApJ, 438, 539

Girardi M., Escalera E., Fadda D., Giuricin G., Mardirossian F., Mezzetti M., 1997, ApJ, 482, 41

Jones C., Forman W., 1999, ApJ, 511, 65

Kelson D. D., Zabludoff A. I., Williams K. A., Trager S. C., Mulchaey J. S., Bolte M., 2002, ApJ, 576, 720

Krempeć-Krygier J., Krygier B., 1999, AcA, 49, 403

Kronawitter A., Saglia R. P., Gerhard O., Bender R., 2000, A\&AS, 144, 53

Madejsky R., 1991, A\&A, 247, 348

Madejsky R., Bender R., Moellenhoff C., 1991, A\&A, 242, 58

Madejsky R., Bien R., 1993, A\&A, 280, 383

Romanowsky A.J., Douglas N.G., Arnaboldi M., Kuijken K., Merrifield M.R., Napolitano N.R., Capaccioli M., Freeman K.C., 2003, Science, 301, Issue 5640, 1696

Saglia R. P., et al., 1993, ApJ, 403, 567

Saglia R. P., Kronawitter A., Gerhard O., Bender R., 2000, AJ, 119, 153

van der Marel R. P., Franx M., 1993, ApJ, 407, 525 